\title{
A mathematical model of traffic segregation on multilane road
}

\author{
Pavel Pospelov ${ }^{1}$, Aleksey Kostsov ${ }^{1}$, Alexander Tatashev ${ }^{1,2}$, Marina Yashina ${ }^{1,2}$ \\ ${ }^{1}$ Moscow Automobile and Road Construction State Technical University (MADI) \\ 64, Leningradsky prospect, Moscow, Russia \\ ${ }^{2}$ Moscow Technical University of Communications and Informatics (MTUCI) \\ 8-a, Aviamotornay street, Moscow, Russia
}

\begin{tabular}{l} 
Article Info \\
\hline Received Dec $21^{\text {th }}, 2018$ \\
\\
\hline Keyword: \\
Traffic segregation. \\
Multilane. \\
Road. \\
Freeway. \\
Capacity. \\
\hline
\end{tabular}

\begin{abstract}
We study a process of segregation of traffic flow on a multilane highway. Our study is based on the stochastic mathematical model developed by Buslaev A.P. et al. in 2006, 2008 years. This model is based on the deterministicstochastic approach that was developed by Buslaev in 2005. In the model, particles, moving on a lattice, correspond to vehicles. We consider the problem of optimization of segregation characteristics. We consider an example of segregation process on five-lane segment of a road. The length of the segment equals 4000 meters. The calibration of the model parameters is based on empirical data obtained in measurements on roads.
\end{abstract}

\section{Corresponding Author:}

\section{Pavel Pospelov,}

Moscow Automobile and Road Construction State Technical University (MADI),

64, Leningradsky prospect, Moscow, Russia

Email: pospelovpi@madi.ru

\section{Introduction}

Currently, the network of main streets and highways, despite the total length of less than $5 \%$ of the total length of the road network, provides up to half of the total mileage of cars. For example, the Federal road system of the United States, with a length of $1.6 \%$ serves $23.8 \%$ of the total mileage, and within the influence of large and largest cities in the United States on high-speed roads account for 30 to 50\% of the mileage, [1] (Mikhailov A., 2004). In Russia, the main road network accounts for 3.2\% of the total length of roads, but performs more than 30\% of freight and passenger traffic (Babkov V., 1993). The huge role of the network of main roads lies in ensuring the quality of transport services to the population.

To highways impose high technical requirements, they are designed to ensure the movement of vehicles in continuous mode with high speeds. Geometrical parameters of the plan, longitudinal and cross-section of segments of highways allow, along with ensuring high speeds and indicators of traffic safety, to provide and the maximum possible indicators of their capacity (Silyanov V., 2008).

In addition to the motorway segments, the most important elements of motorways, which often determine their capacity, are the merge and diverge areas of freeway interchange - interchange influence areas (Fig.1). 

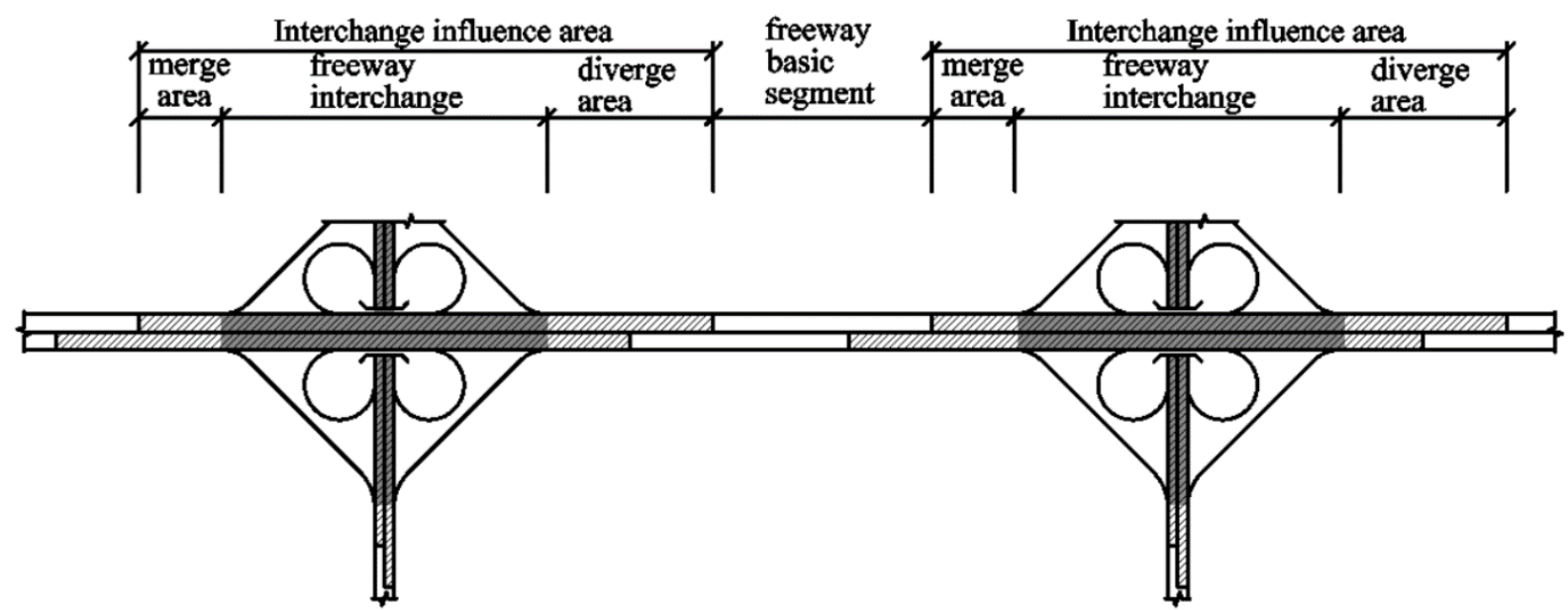

Fig. 1 Freeway interchange influence areas

The influence of the mode of operation of such sections on the capacity of highways is due to the process of interaction of the traffic flow leaving the highway (or entering from it) with the transit traffic flow moving along the highway. In order to develop recommendations for determining the capacity of highways, the article proposes a stochastic model of segregation of traffic flows. The particles move in the same direction over a multi-lane cell field. The waste stream occurs at a transit station. There are two types of particles. Particles of the first type move along one strip and are not inclined to move to another. Each particle of the second type tends to move sequentially to the far right band. The length of the section and the intensity of flows on the lanes at the beginning of the section are given. We develop an approach to calculate the probability that a fixed particle of the second type will pass sequentially along the extreme right band on the site. Our approach is based on the version of the flow segregation model developed in [4] (Bugaev A. et al., 2006), [5] (Bugaev A. et al., 2008), and we use the deterministic-stochastic approach developed in [6] (Buslaev A. et al., 2003), [7] (Buslaev A. et al., 2005). We consider a stochastic model of flow segregation. Particles move in the same direction on multi-lane field of cells. Flow departing occurs on a transit section. There are two types of particles. Particles of the first type move along the same lane and do not tend to transit to another lane. Each particle of the second type tends to transit successively to the utmost right lane. The length of the section and the intensities of flows on the lanes in the section beginning are given. We develop an approach to calculate the probability that a fixed particle of the second type will transit successively to the utmost right lane on the section.

\section{Description of the model}

Assume that particles of two types move on $K$-lane section. The section is developed into $M$ segments. Each segments contains $l$ cells. The size (length) of each cell, located on the segment $m$, equals $d_{m}, m=1, \ldots, M$. The coordinates of a cell are $(x, y)$, where $x$ is the index of the lane, and $y$ is the index of the point. The $m$-th segment of the $k$ th lane contains the cells:

$$
(i, y), y=(j-1) l+1, \ldots, j l \text {. }
$$

There are two types of particles. Particles of the first type move along the same lane and do not tend to transit to another lane. Each particle of the second type tends to transit successively to the $K$ th lane. The particle of the first type correspond to cars that do not tend to change lanes. The particles of the second type correspond to particles that intend to move along another road after passing the section. The value of $d$ corresponds to the dynamical dimension of cars.

The rule of particles movement on the segment $m, m=1, \ldots, M$.

(1) If the particle of the first type is on the lane $x$, then it is on this lane while it moves along the section. For a time quantum $\Delta t$, the particle of the first type, located on the lane $k$ and the segment $m$ with probability $\lambda_{1, m} \Delta t$ tends to move onto one cell in the direction of movement. The attempt is realized if the cell ahead is vacant. 
(2) If the particle of the second type is in the cell $(k, j), k<L$, and the cell $(k+1, j)$ (a cell on the lane to the right of the particle) is vacant, then the particle comes to the cell $(k+1, j)$ with probability $\lambda_{2, m} \Delta t$.

(3) If a particle of the second type is on the lane $k$, and the cell, located ahead this particle is vacant, and the neighboring cell on the lane $\mathrm{k}$, is occupied, then the particle does not move.

Assume that, at time $s \Delta t$, the particle of the second type is on the m-th segment, on the $k$-th lane in the cell $(k, m), k=1, \ldots, K-1$. Suppose $p(k, m)$ is the probability that this particle will be in the cell

\section{Calculation of traffic flow characteristic}

Let's make the following assumptions: in each cell located on the $m$-th segment on the $k$-th lane, with probability $r_{i}(k, m)$ there is a particle of type $i, i=1,2, i=1, \ldots, K, m=1, \ldots, M$. With probability $1-$ $r(k, m)$, where $r(k, m)=r_{1}(k, m)+r_{2}(k, m)$, this cell is free. Probabilities of states do not depend on each other. Formula for $p(k, m)(k=1, \ldots, K-1, m=1, \ldots, M)$ :

$$
p(k, m)=(1-r(k+1, m)) \lambda_{2, m} \Delta t .
$$

Suppose $(k, m)$ is the density of flow on the lane $k$ of the segment $m$. Assume that

$$
r_{i}(k, m)=d_{m} x_{i}((k, m) k=1, \ldots, K, m=1, \ldots, M ., i=1,2 .
$$

Flow densities for the first segment, i.e. the values of $\rho_{i}(k, 1), s=1,2, k=1, \ldots, K$, are specified.

Let $\beta(k, m)$ is the probability that a particle of the second type that started moving along the $m$-th segment on the $k$-th lane, $k=1, \ldots, K-1, m=1, \ldots, M$, will have time before the end of the passage of this segment to move to the adjacent right lane.

The values of $q_{i}(k, 1)$ of the intensities of the flow of particles moving along the $k$-th lane on the first segment are calculated by the formula $(i=1,2)$

$$
\begin{aligned}
q_{1}(k, 1)\left(v_{1}+(1-r(k, 1))\right. & \left.\lambda_{1, m}, \Delta t\right), k=1, \ldots, K . \\
& q_{2}(k, 1)=x_{2}(k, 1) v_{1}, k=1,, K .
\end{aligned}
$$

In accordance with the law of conservation of intensity

$$
q_{1}(k, m)=q_{1}(k, 1), m=2,3, \ldots, M .
$$

Intensity $q_{i}(k, m)$ of the second type particle flow on the $k$-th lane on the $m$-th segment, $m=2,3, \ldots, M$ is calculated according to the formulas

$$
\begin{gathered}
q_{2}(1, m)=q_{2}(1, m-1)(1-\beta(1, m-1)) \\
q_{2}(k, m)=q_{2}(k-1, m-1) \beta(k, m-1)+q_{2}(k, m)(1-\beta(k, m)) \\
q_{2}(K, m)=q_{2}(K-1, m-1) \beta(K-1, m-1)+q_{2}(K, m)
\end{gathered}
$$

The approximate values of probabilities that the cells of the lane $k=1, \ldots, K$ and segment $m=2, \ldots, M$ are occupied by particles of a given type are found from the relations

$$
\begin{gathered}
q_{1}(k, m)=\rho_{1}(k, m)\left(v_{1}+(1-r(k, m)) \lambda_{1, m} \Delta t\right), k=1, \ldots, K, m=1, \ldots, M . \\
q_{2}(k, m)=\rho_{2}(k, m) v_{m}, m=1, \ldots, M . \\
r(k, m)=r_{1}(k, m)+r_{2}(k, m) \leq 1
\end{gathered}
$$

This system of equations for each pair of values $k, m$ is reduced to a square equation. If the system has more than one solution, then take the smaller value $\rho_{2}(k, m)$. Let $k, m$ be a random variable representing the average time before a particle transitions to the $k+1$ st lane under the assumption that the length of the segment is not limited. Let $\eta(k, m)$ be a random variable distributed according to the normal law with the corresponding parameters

$$
\beta(k, m)=0,5+\Phi\left(\frac{L_{m}-a(k, m)}{\sigma(k, m)}\right), k=1, \ldots, K, m=1, \ldots, M-1
$$

where $\Phi(x)$ is the Laplace function:

$$
\begin{gathered}
\Phi(x)=\frac{1}{\sqrt{2 \pi}} \int_{0}^{x} e^{-\frac{z^{2}}{2}} d z, \\
a(k, m)=\frac{v_{m} \Delta t}{p(i, 1)}
\end{gathered}
$$




$$
\sigma^{2}(i, m)=\frac{p(i, m)\left(v_{m} \Delta t\right)^{2}}{(1-p(i, m))^{2}}
$$

Suppose $\alpha$ is the probability that a particle transits to the utmost right lane successively is equal to

$$
\alpha=\left(q_{2}(K, M)+\beta(K-1, M-1) q_{2}(K-1, M-1)\right) / \sum_{k=1}^{K} q_{2}(k, 1) .
$$

Calibration of the model parameters was carried out on the basis of data obtained as a result of road experiments. Segregation in a 4000 meter long section was considered. In the model, the site was divided into 40 segments each 100 meters long. Values of flow intensities on different lanes and segments are represented in Table 1.

Table 1. Dependence of flow intensity $q_{2}(1 / \mathrm{sec})$ on the road lane and segment, $K=5, M=40$, tsec, $L_{m}=100 \mathrm{~m}$,

\begin{tabular}{|c|c|c|c|c|c|c|c|c|}
\hline $\begin{array}{c}\text { Number of lane } \\
\text { ISegment number }\end{array}$ & 1 & 2 & 3 & 4 & 5 & 6 & 7 & 8 \\
\hline 1 & 0,060 & 0,046 & 0,035 & 0,027 & 0,021 & 0,016 & 0,012 & 0,009 \\
\hline 2 & 0,060 & 0,060 & 0,057 & 0,052 & 0,046 & 0,040 & 0,034 & 0,029 \\
\hline 3 & 0,060 & 0,060 & 0,060 & 0,059 & 0,058 & 0,055 & 0,051 & 0,047 \\
\hline 4 & 0,060 & 0,060 & 0,060 & 0,060 & 0,061 & 0,061 & 0,060 & 0,060 \\
\hline 5 & 0,060 & 0,074 & 0,088 & 0,101 & 0,115 & 0,128 & 0,142 & 0,155 \\
\hline $\begin{array}{l}\text { Number of road lane } \\
\text { ISegment number }\end{array}$ & 9 & 10 & 11 & 12 & 13 & 14 & 15 & 16 \\
\hline 1 & 0,007 & 0,005 & 0,004 & 0,003 & 0,002 & 0,002 & 0,001 & 0,001 \\
\hline 2 & 0,024 & 0,020 & 0,017 & 0,014 & 0,011 & 0,009 & 0,007 & 0,006 \\
\hline 3 & 0,043 & 0,039 & 0,034 & 0,030 & 0,026 & 0,023 & 0,019 & 0,016 \\
\hline 4 & 0,058 & 0,056 & 0,054 & 0,051 & 0,048 & 0,045 & 0,042 & 0,038 \\
\hline 5 & 0,167 & 0,179 & 0,191 & 0,202 & 0,212 & 0,222 & 0,231 & 0,239 \\
\hline $\begin{array}{l}\text { Number of road lane } \\
\text { ISegment number }\end{array}$ & 17 & 18 & 19 & 20 & 21 & 22 & 23 & 24 \\
\hline 1 & 0,001 & 0,001 & 0,000 & 0,000 & 0,000 & 0,000 & 0,000 & 0,000 \\
\hline 2 & 0,005 & 0,004 & 0,003 & 0,002 & 0,002 & 0,001 & 0,001 & 0,001 \\
\hline 3 & 0,014 & 0,012 & 0,010 & 0,008 & 0,007 & 0,005 & 0,004 & 0,004 \\
\hline 4 & 0,035 & 0,032 & 0,028 & 0,025 & 0,023 & 0,020 & 0,018 & 0,016 \\
\hline 5 & 0,246 & 0,253 & 0,259 & 0,264 & 0,269 & 0,273 & 0,277 & 0,280 \\
\hline $\begin{array}{l}\text { Number of road lane } \\
\text { ISegment number }\end{array}$ & 25 & 26 & 27 & 28 & 29 & 30 & 31 & 32 \\
\hline 1 & 0,000 & 0,000 & 0,000 & 0,000 & 0,000 & 0,000 & 0,000 & 0,000 \\
\hline 2 & 0,001 & 0,001 & 0,000 & 0,000 & 0,000 & 0,000 & 0,000 & 0,000 \\
\hline 3 & 0,003 & 0,002 & 0,002 & 0,002 & 0,001 & 0,001 & 0,001 & 0,001 \\
\hline 4 & 0,014 & 0,012 & 0,010 & 0,009 & 0,008 & 0,007 & 0,006 & 0,005 \\
\hline 5 & 0,283 & 0,285 & 0,287 & 0,289 & 0,291 & 0,292 & 0,293 & 0,294 \\
\hline $\begin{array}{l}\text { Number of road lane } \\
\text { ISegment number }\end{array}$ & 33 & 34 & 35 & 36 & 37 & 38 & 39 & 40 \\
\hline 1 & 0,000 & 0,000 & 0,000 & 0,000 & 0,000 & 0,000 & 0,000 & 0,000 \\
\hline 2 & 0,000 & 0,000 & 0,000 & 0,000 & 0,000 & 0,000 & 0,000 & 0,000 \\
\hline 3 & 0,001 & 0,000 & 0,000 & 0,000 & 0,000 & 0,000 & 0,000 & 0,000 \\
\hline 4 & 0,004 & 0,003 & 0,003 & 0,003 & 0,002 & 0,002 & 0,002 & 0,001 \\
\hline 5 & 0,295 & 0,296 & 0,297 & 0,297 & 0,298 & 0,298 & 0,298 & 0,299 \\
\hline
\end{tabular}
${ }_{m}=0.151 / \mathrm{sec}, \quad d_{m}=25 \mathrm{~m}, v_{m}=10 \mathrm{~m} / \mathrm{sec}, m=1, \ldots, 40$ 


\section{Conclusion}

An approach for calculating the characteristics of traffic flow segregation based on the stochastic segregation model is developed. Deterministic-stochastic approach to traffic flow analysis is used. The presented studies can be used to determine the capacity of traffic segregation sections, which will improve the validity of design decisions in the design of highways, which in turn will contribute to improving the quality of transport services.

\section{ACKNOWLEDGEMENTS}

This work has been supported by the Russian Foundation for Basic Research, Grant

No. 17-01-00821-a and Grant No. 17-29-03419-a.

\section{REFERENCES}

[1] Mikhailov A., Golovnykh I. Modern trends in the design and reconstruction of urban road networks, Novosibirsk. Nauka. $2004-267$ p.

[2] Babkov V. Road conditions and traffic safety: Moscow, Transport, 1993.- $271 \mathrm{p}$.

[3] Silyanov V., Domke E. Transport and operational quality of roads and city streets: Moscow, Akademiya, 2008. - 352 p.

[4] Buslaev A. P., Novikov A. V., Prikhodko V. M., Tatashev A. G., Yashina M.V. Probabilistic and imitation approaches to optimization of road traffic. Moscow, Mir, 2003. - 368 p.

[5] Buslaev A. P., Prikhodko V.M., Tatashev A.G., Yashina M.V. The deterministic-stochastic flow model, 2005. arXiv:physics/0504139[physics.sos-ph], pp. $1-21$.

[6] Silyanov V., Domke E. Transport and operational quality of roads and city streets: Moscow, Akademiya, 2008. - 352-4.

[7] Bugaev A. S., Buslaev A. P., Tatashev A. G. Monotone random movement of particles on a nintegernumber-lane and LYuMEN problem. Mathematicheskoye Modelirovaniye, 2006, 18(12), pp. 19 - 34.

[8] Bugaev A. S., Buslaev A. P., Tatashev A. G. Modeling of segregation of two-lane flow particles. Matematicheskoye Modelirovaniye, 2008, 10(9), pp. 111-119 